\title{
Impact of chronic intermittent external compressions on forearm blood flow capacity in humans
}

\author{
Bruno T. Roseguini, \\ Department of Biomedical Sciences, University of Missouri, Columbia, MO, USA \\ Ryan Sheldon, \\ Department of Health and Kinesiology, Purdue University, Lambert Fieldhouse, 800 W. Stadium \\ Ave., West Lafayette, IN 47907, USA
}

Abigail Stroup,

Department of Health and Kinesiology, Purdue University, Lambert Fieldhouse, 800 W. Stadium Ave., West Lafayette, IN 47907, USA

Jeffrey W. Bell, Department of Health and Kinesiology, Purdue University, Lambert Fieldhouse, 800 W. Stadium Ave., West Lafayette, IN 47907, USA

David Maurer, Department of Health and Kinesiology, Purdue University, Lambert Fieldhouse, 800 W. Stadium Ave., West Lafayette, IN 47907, USA

\author{
Brett D. Crist, \\ Department of Orthopaedic Surgery, University of Missouri, Columbia, MO, USA
}

M. H. Laughlin, and Department of Biomedical Sciences, University of Missouri, Columbia, MO, USA

Sean C. Newcomer

Department of Health and Kinesiology, Purdue University, Lambert Fieldhouse, 800 W. Stadium Ave., West Lafayette, IN 47907, USA

\section{Abstract}

\begin{abstract}
During dynamic exercise, the vasculature embedded within skeletal muscle intermittently collapses due to increased intramuscular pressure (IMP). The aim of this study was to ascertain whether oscillations in IMP during muscle contractions independently contribute to exercise training-induced increases in blood flow capacity (BFC). Based on IMP measurements during handgrip exercise, we attempted to mimic the action of repeated vascular compressions by using external inflatable cuffs. Thus, 24 healthy young male subjects underwent a 4-week program (5 days/week, $1 \mathrm{~h}$ /day) of application of external compressions of the non-dominant forearm, while the dominant limb served as an internal control. To evaluate the impact of compression pressures of different magnitudes, subjects were randomly assigned to one of three groups: 50, 100 and 150 $\mathrm{mmHg}$ of external compression. Prior to the intervention and after 2 and 4 weeks of treatment, we measured peak forearm blood flow (PBF) (Doppler ultrasound) and calculated peak vascular
\end{abstract}

(C) Springer-Verlag 2010

snewcome@purdue.edu .

Electronic supplementary material The online version of this article (doi: 10.1007/s00421-010-1657-6) contains supplementary material, which is available to authorized users.

Conflict of interest None. 
conductance (PVC) following $10 \mathrm{~min}$ of forearm ischemia. In the 50 and $100 \mathrm{mmHg}$ groups, application of intermittent compressions did not alter PBF in either control or intervention forearms. In the $150 \mathrm{mmHg}$ group, there was a trend $(P=0.04)$ for greater increases in PBF from baseline after 4 weeks in the intervention forearm compared to the control forearm (delta PBF: 4.2 \pm 2.5 vs. $-2.1 \pm 2.0\left(\mathrm{ml}(100 \mathrm{ml})^{-1} \mathrm{~min}^{-1}\right)$, in the intervention and control forearms, respectively), but the changes in PVC were not significant $(P=0.1)$. These findings suggest that repeated oscillations in IMP contribute minimally to exercise-induced increase in forearm BFC in healthy young humans.

\section{Keywords}

Skeletal muscle; Blood flow capacity; Exercise training

\section{Introduction}

Exercise training promotes a number of adaptations in the skeletal muscle vasculature including increased capillarization, enlargement and growth of new arterioles and accompanying changes in vasomotor reactivity (Laughlin and Roseguini 2008). Combined, these structural and functional changes result in an increased capacity of the vasculature to accommodate flow (Laughlin and Ripperger 1987; Laughlin and Roseguini 2008). The results from studies employing small muscle mass exercise demonstrate that training for as little as 4 weeks can enhance limb blood flow capacity (BFC), as estimated from the peak blood flow (PBF) response to a period of ischemia (i.e., reactive hyperemia) (Alomari et al. 2001; Green et al. 1994; Sinoway et al. 1987). Likewise, cross-sectional studies comparing trained and sedentary individuals clearly show a strong relation between training status and PBF following limb occlusion (Kroese 1977; Reading et al. 1993; Snell et al. 1987). Interestingly, despite the widespread recognition of the importance of vascular remodeling during exercise training (Saltin 1977), the signals and mechanisms that initiate and mediate these adaptations remain poorly understood.

The vasculature embedded within the skeletal muscle is strongly tethered to the surrounding tissue, and during normal contractile activity these vessels are subjected to multiple mechanical forces, including external compression (Gray et al. 1967; Hudlicka 1998). Indeed, the intramuscular pressure (IMP) is known to increase profoundly even during mild contractions, causing the vasculature to intermittently collapse and blood flow and shear stress to oscillate (Barcroft and Millen 1939; Hirche et al. 1970; Radegran and Saltin 1998). Studies that used inflatable cuffs to mimic the action of contraction-induced oscillations in IMP advanced the notion that this mechanism can evoke vasodilation (Kirby et al. 2007) and reduce regional arterial stiffness (Heffernan et al. 2007). Notably, emerging evidence also implicates repeated mechanical compressions as a possible participant in vascular remodeling in skeletal muscle. Chen and et al. first showed in rodents that application of external compressions to the lower limb can up-regulate eNOS expression in skeletal muscle (Chen et al. 2002). Using a similar approach, we recently observed that intermittent compressions increase mRNA expression of vascular endothelial growth factor (VEGF) and monocyte chemoattractant protein-1 (MCP-1), two key players in the angiogenic process in skeletal muscle (Roseguini et al. 2010). Accordingly, repeated mechanical compressions during muscle contraction may trigger increased expression of growth factors and angiogenic mediators and therefore contribute to vascular remodeling in skeletal muscle. To date, however, the independent role of mechanical compressions as signals for vascular remodeling in skeletal muscle remains unknown. 
To gain insights into this issue, we determined the longterm impact of external mechanical compressions on BFC in humans. We reasoned that application of external mechanical compression of the forearm, and the consequent oscillations in IMP, could promote vascular remodeling and thus enhance BFC. To properly mimic the pressures experienced by the vasculature during exercise, we first determined the force-IMP relationship in the forearm flexor muscles. Based on these measured pressures, we investigated the impact that 4 weeks of daily external mechanical compressions has on maximal blood flow responses to prolonged ischemia. We hypothesized that the magnitude of change in peak reactive hyperemic blood flow following daily external mechanical compressions would be graded, such that higher external compression pressures would cause more robust changes in forearm BFC.

\section{Methods}

\section{Subjects}

Experiments were performed in seven male subjects in study 1 (age: $31 \pm 2$ years and body weight: $71 \pm 3 \mathrm{~kg}$ ) and 24 male subjects in study 2 (age: $23 \pm 1$ years and body weight: $81 \pm$ $3 \mathrm{~kg}$ ). All individuals were initially asked to complete a medical and exercise history form and received detailed instructions about the protocol. Subjects were excluded if they were smokers, hypertensive (resting blood pressure $>140 / 90 \mathrm{mmHg}$ ), displayed any evidence of cardiovascular, pulmonary and metabolic disease, and if they were on any medications. To avoid the confounding effect of baseline training status, subjects who participated in any kind of physical activity in which the forearm was predominantly involved (tennis, climbing, strength training, etc.) were also excluded. All procedures were approved by the University of Missouri Health Sciences and Purdue University Institutional Review Boards and subjects gave their informed written consent prior to participation in the experiment.

\section{Study design}

The experimental design comprised two studies. In study 1, the IMP developed in the forearm due to handgrip exercise and external mechanical compressions were determined. These IMP measurements were used to establish three experimental pressure groups (50, $100,150 \mathrm{mmHg}$ ) into which subjects were randomly assigned to undergo a 4-week program of intermittent external forearm compressions ( 5 days/week, $1 \mathrm{~h}$ ). Forearm BFC, as estimated by PBF and peak vascular conductance (PVC) responses following $10 \mathrm{~min}$ of forearm occlusion, was determined at baseline and following 2 and 4 weeks of daily external mechanical compressions. Additionally, brachial artery blood flow and shear patterns were determined during a single session of intermittent compressions to characterize the acute effects of application of mechanical compressions.

\section{Intramuscular pressure measurements}

Intramuscular pressure was measured using a fluid-filled catheter (gauge 16 and 18) connected to a pressure transducer interfaced with a monitor. Under sterile conditions, the catheter was inserted into the superficial anterior compartment of the forearm at an angle of $20-40^{\circ}$, advanced for $1-1.5 \mathrm{~cm}$ within the muscle toward the elbow and covered with tape. Subjects were then asked to complete two protocols to determine the IMP during handgrip exercise and external application of pressure of various intensities. Initially, subjects performed three maximal handgrip contractions with the non-dominant limb to determine the maximal voluntary contraction (MVC) and maximal IMP. Subsequently, IMP was determined during sustained contractions $(\sim 10 \mathrm{~s})$ performed at different exercise intensities ( $5 \mathrm{~kg}$ increments) in random order up to maximum. The handgrip device (Hand Dynamometer, Stoeling, Wood Dale, IL) was directly connected to a data acquisition system 
(Powerlab, AD Instruments, Colorado Springs, CO) and visual feedback of force development was given to the subjects on the computer screen.

To determine the relation between IMP and externally applied compressions, a small cuff $(11 \times 85 \mathrm{~cm}, \mathrm{SC10}$, Hokanson, Bellevue, WA $)$ was firmly wrapped around the forearm, above the location of the intramuscular catheter. The cuff was rapidly inflated to several different pressures in random order (10-200 $\mathrm{mmHg}$ ) at $10 \mathrm{mmHg}$ increments (Hokanson E20 and AG101, Bellevue, WA). IMP was recorded 10-15 s following cuff inflation.

\section{Application of external mechanical compressions}

Determination of IMP revealed that the forearm vasculature is exposed to a wide range of pressures during moderate-to-high intensity handgrip exercise (Fig. 1). It is worth mentioning that the pressures reported herein reflect only the range experienced by superficial tissues in the forearm. As reported previously in several muscles of the lower limb (Sejersted and Hargens 1995; Sejersted et al. 1984), IMP varies pronouncedly between subjects at similar absolute loads and increases linearly with muscle depth (see the section "Experimental considerations"). Therefore, three different pressures generated by mechanical compression $(50,100$ and $150 \mathrm{mmHg}$ ) were utilized to mimic the whole range of pressures produced during exercise in the forearm. Twenty-four subjects were randomly allocated to one of the three compression groups and underwent a 4-week program of external forearm applications ( 5 days/week for $1 \mathrm{~h}$ ). In each session, the subjects were instrumented with two cuffs (SC10/SC12, Hokanson, Bellevue, WA) wrapped around the entire experimental forearm (non-dominant) that was inflated/deflated cyclically $(2 \mathrm{~s} \mathrm{on} / 3 \mathrm{~s}$ off) with a rapid cuff inflator unit (Hokanson E20, Bellevue, WA), while the dominant forearm served as an internal control and was not exposed to external compressions. This frequency of compressions (12 compressions/min) was selected based on the study of Sinoway et al. (1987). A computer-generated signal (PowerLab 4/S system, AD Instruments) allowed for application of compression rates specified above. Before, halfway through ( 2 weeks) and at the end (4 weeks) of the intervention, all subjects completed a measurement session that included anthropometric measurements and determination of brachial blood flow following $10 \mathrm{~min}$ of forearm ischemia. Prior to the measurement sessions, subjects fasted and avoided caffeine for $4 \mathrm{~h}$ and refrained from strenuous physical activity for $12 \mathrm{~h}$.

\section{Anthropometric and handgrip force measurements}

After recording height and body weight with a conventional scale, forearm volume was determined from the head of the ulna to the olecranon process using water displacement (Newcomer et al. 2004). MVC was determined using a handgrip dynamometer (Stoeling, Wood Dale, IL) interfaced with a data acquisition system (Powerlab, AD Instruments, Colorado Springs, CO). MVC was determined as the highest force achieved on three attempts.

\section{Brachial artery blood flow determination and reactive hyperemia}

After obtaining anthropometric measures, subjects were positioned supine in a dark, climatecontrolled $\left(22-25^{\circ} \mathrm{C}\right)$ quiet room and instrumented for the study. This included a rapidinflation/deflation pneumatic cuff placed around the forearm (Hokanson, Bellevue, WA), a conventional cuff in the contralateral arm for automated determination of blood pressure (Tango+, SunTech Medical, Morrisville, NC) with a 3-lead (ECG) for heart rate measurement. Blood flow through the brachial artery was determined with Doppler ultrasound as previously described (Padilla et al. 2009). Briefly, the brachial artery was imaged $2-5 \mathrm{~cm}$ above the antecubital fossa with a 5-12 MHz multifrequency linear-array transducer attached to a two-dimensional high-resolution ultrasound system (Terason 
T3000; Teratech, Burlington, MA). Doppler velocity was determined using an insonation angle of $60^{\circ}$ with the sample volume being maximized in an attempt to minimize overestimation of mean blood velocity (MBV). The probe position relative to the antecubital fossa was recorded for repeat sessions. After $20 \mathrm{~min}$ of rest, brachial artery diameter and flow velocity were recorded continuously for $1 \mathrm{~min}$. The cuff located in the forearm was then rapidly inflated to $220 \mathrm{mmHg}$ and the occlusion was sustained for $10 \mathrm{~min}$. The transducer was held in place during forearm occlusion by a clamp to insure consistency of image location from pre- to post-inflation. At $30 \mathrm{~s}$ before cuff deflation, diameter and flow velocity recordings resumed and continued for $3 \mathrm{~min}$ thereafter. Blood pressure and heart rate were also recorded at baseline and 1 min before cuff deflation. After completion, the same procedure was repeated in the other forearm. The order of the experiments was randomized. The same experienced sonographer performed all reactive hyperemic measurements in the study.

\section{Determination of brachial artery shear stimulus during mechanical compressions}

To characterize the shear rate profiles during externally applied compressions, brachial artery diameter and velocity were also measured during a single compression session in all three experimental groups. Subjects sat comfortably with both forearms and hands resting on a table with the non-dominant forearm instrumented with two cuffs as described above. Brachial artery blood flow and diameter were continuously measured for $30 \mathrm{~s}$ at baseline and during the compressions at each subject's prescribed compression pressure.

\section{Data analysis}

Brachial artery diameter before and after reactive hyperemia were determined using edgedetection software (Brachial Analyzer; Medical Imaging Applications, Coralville, IA) (Padilla et al. 2009). A customized MatLab program was used for blood velocity determinations for each cardiac cycle as described previously (Padilla et al. 2010). Brachial artery blood flow $(\mathrm{BF})$ was calculated as:

$$
\mathrm{BF}=\mathrm{MBV} \times \pi(d / 2)^{2} \times 60,
$$

where the BF is forearm blood flow in $\mathrm{ml} \mathrm{min}^{-1}$, the MBV is in $\mathrm{cm} \mathrm{s}^{-1}, d$ is the brachial diameter in $\mathrm{cm}$, and 60 is used to convert from $\mathrm{ml} \mathrm{s}^{-1}$ to $\mathrm{ml} \mathrm{min}^{-1}$. Blood flow was corrected for forearm volume. Vascular conductance was calculated as $(\mathrm{BF} / \mathrm{MAP}) \times 100$, where MAP is mean arterial pressure obtained $1 \mathrm{~min}$ prior to cuff release. The automated blood pressure monitor employed did not allow for the continuous measurement of blood pressure during reactive hyperemia. Accordingly, it should be emphasized that the blood pressure values used in the calculation of vascular conductance might not reflect the fast, albeit minor, changes in BP that could have occurred during the early phase of hyperemia.

Peak blood flow post-occlusion (PBF) was calculated from the average of the two highest MBV that occurred simultaneously following cuff release. This occurred within the first $10 \mathrm{~s}$ following cuff release. The blood flow area under the curve of the reactive hyperemic response $\left(\mathrm{AUC}_{\mathrm{RH}}\right)$ was calculated from the point of cuff deflation to $270 \mathrm{~s}$. Brachial artery flow-mediated dilation (FMD) was determined as the peak diameter following cuff release and was represented as a percentage change from baseline. Shear rate during application of compressions was defined as $4 \times V_{\mathrm{m}} / D$, where $V_{\mathrm{m}}$ is $\mathrm{MBV}$ and $D$ is the arterial diameter (Newcomer et al. 2008; Padilla et al. 2009). FMD was normalized to the shear rate area under the curve $\left(\mathrm{AUC}_{\mathrm{SR}} ; \mathrm{s}^{-1} \mathrm{~s}\right)$, calculated from the point of cuff deflation to the maximum post-deflation diameter (Padilla et al. 2009). Antegrade and retrograde peak and mean blood velocities were used to calculate antegrade and retrograde shear rates. The oscillatory shear 
index (OSI) was calculated as: |retrograde shear|/(|antegrade shear|+ |retrograde shear|) (Newcomer et al. 2008; Padilla et al. 2010).

\section{Statistical analysis}

Anthropometric characteristics and grip strength before and after the intervention and shear rate profiles during compressions were compared between groups by an ANOVA for repeated measures. The impact of 4 weeks of external compressions on hemodynamic responses in both forearms was compared using a mixed procedure in SAS v9 (SAS Institute Inc., Cary, NC, USA). A similar approach was used to compare the delta changes in FBF and FVC from the pre-intervention values. In view of the large number of pairwise comparisons being made, the comparison-wise error rate was controlled at the 0.01 level. This choice is less conservative than using a Bonferroni adjustment. All data are presented as mean \pm SE.

\section{Results \\ IMP during exercise and external compression}

As expected (Sejersted and Hargens 1995; Sejersted et al. 1984), the relation between contraction force and IMP was linear $(r=0.94)$ (Fig. 1a), but the slopes varied substantially between individuals. Likewise, IMP increased proportionally to the external pressure during cuff inflations $(r=0.99)$ (Fig. 1b).

\section{Subject characteristics}

Anthropometric characteristics and grip strength of subjects involved in study 2 are shown in Table 1. No significant changes were observed between the three groups before and after 4 weeks of intervention.

\section{Shear rate profile during compressions}

Mean and peak shear rates in the brachial artery before and during application of compressions are shown in Table 2. As expected, intermittent cuff inflation/deflation produced a fluctuating shear rate profile, evidenced by the significant increase in OSI in all three groups (Table 2). Rapid cuff inflation evoked a pressure-dependent increase in retrograde flow followed by a modest, non-significant increase in antegrade flow and shear rates during cuff deflation (Table 2; supplemental Fig. 1). In spite of the changes in oscillatory profile, mean shear rate was not different compared to baseline in all three groups (Table 2).

\section{Reactive hyperemia responses}

The impact of 4 weeks of external forearm compressions on hemodynamic responses to prolonged forearm ischemia is shown in Tables 3 and 4 . No baseline differences were detected between groups for all variables measured. Brachial artery hemodynamics was also comparable between the dominant and non-dominant forearms in all groups before the intervention. Contrary to our hypothesis, there was no significant interaction between group $(50,100,150 \mathrm{mmHg}), \operatorname{limb}$ (dominant/nondominant) and time of evaluation (baseline, 2 weeks and 4 weeks) for brachial artery hemodynamic responses to ischemia $(P=0.6)$. When correcting for the baseline values (pre-intervention), PBF and PVC responses to occlusion showed a distinct profile among groups throughout the 4 weeks. In both the 50 and 100 $\mathrm{mmHg}$ groups, PBF decreased slightly in both control and intervention forearms ( 18\%) after 4 weeks when compared with baseline (Fig. 2). In contrast, in the $150 \mathrm{mmHg}$ group, PBF increased non-significantly in the compressed limb after 2 weeks (by 16\%) and 4 weeks (by 20\%) when compared with baseline, while in the control forearm this variable 
increased only slightly at 2 weeks (5\%) and decreased by $10 \%$ at 4 weeks. The comparison of the delta changes from baseline (delta PBF) between limbs at 4 weeks in this group revealed a trend for increased $\mathrm{BFC}$ in the forearm that underwent compressions $(P=0.04$, Fig. 2c). However, delta PVC responses in the dominant and non-dominant limbs were not statistically significant in this group ( $P=0.15$, Fig. $3 c)$. Brachial artery FMD did not change significantly following the intervention in all three pressure groups (Tables 3,4 ).

\section{Discussion}

The present study sought to determine whether 4 weeks of application of external forearm compressions of various intensities would enhance forearm peak reactive hyperemic blood flow in healthy young males. The primary new finding was that long-term mechanical deformations produced by external compressions did not evoke important changes in forearm vascular transport capacity in humans. Indeed, although we observed a clear trend for increased PBF throughout the 4 weeks of intervention in the group exposed to compression pressures of $150 \mathrm{mmHg}$, the changes in PVC were not statistically significant. These results support the notion that, at least in the forearm, mechanical factors contribute minimally to the vascular adaptations associated with exercise training.

\section{Experimental considerations}

Muscle contractions activate a number of physiological processes that can trigger and/or modulate vascular remodeling, including increases in muscle metabolism, release and accumulation of metabolites and mechanical factors (Brown and Hudlicka 2003; HansenSmith et al. 2001; Prior et al. 2003, 2004). To determine the potential contribution of intermittent mechanical deformations to the vascular changes evoked by exercise training without the confounding action of other factors, our strategy was to externally apply cyclic mechanical compressions to the forearm with conventional blood pressure cuffs. This approach has been widely used to isolate and study the mechanical influences of contraction on muscle blood flow regulation (Kirby et al. 2007; Lind and Williams 1979; Pyke et al. 2008; Reneman et al. 1980). Although it seems improbable that this strategy perfectly mimics the complex interactions between the vasculature and the muscle tissue during contractions (Gray et al. 1967), our data suggest that externally applied pressures are accurately transmitted to the tissue. Indeed, for the wide range of pressures tested, there was a strong correlation between external pressure and IMP (Fig. 1b). Thus, at least in the superficial compartment of the forearm, changes in IMP analogous to those seen during contraction, can be artificially induced by external pressure application.

The major premise implicit in the design of this study is that the pressures applied externally in the forearm are within the range normally generated by the muscles during contractions. Unfortunately, we were unable to find studies that documented the response of IMP during handgrip exercise in humans. As a preliminary step, we determined the relation between IMP and contraction force in the superficial compartment of the forearm of healthy, young individuals (study 1). A limitation of these experiments was that IMP measurements were only performed in the superficial compartment of the forearm and therefore did not fully represent pressures experienced by deeper tissues. It is well known that IMP during exercise increases linearly with muscle depth, increasing by almost twofold for every centimeter advanced toward the bone (Sejersted and Hargens 1995). To overcome this problem and gain insights into the potential impact of pressure magnitude on the vascular adaptations to external compressions, we chose to apply three different compression pressures $(50,100$ and $150 \mathrm{mmHg}$ ). According to our predictions, and the observed IMP in forearm superficial muscles, this pressure range lies within the physiological spectrum experienced by the forearm vasculature during moderate-to-intense handgrip exercise in young males. Nonetheless, given the expressive heterogeneity in IMP between and within subjects, it is 
impossible to exclude the possibility that, at least in some individuals, higher pressures than the ones employed here are observed during exercise of moderate intensity. Future studies are thus needed to characterize the impact of higher compression pressures on the vasculature.

\section{Vascular remodeling and exercise training}

Traditionally, small muscle mass paradigms have been employed to characterize changes in vascular transport capacity with exercise training in humans (Green 2009; Green et al. 1994; Sinoway et al. 1987). Since central hemodynamics are not strongly perturbed during localized exercise, this model offers a unique opportunity to understand the importance of local mechanisms to traininginduced changes in vascular reactivity and structure (Sinoway et al. 1987). Studies that utilized short-term handgrip exercise training (4 weeks) have shown that PBF following ischemia increases modestly (Alomari et al. 2001; Green et al. 1994; Sinoway et al. 1987). By using a similar approach, our intention in the present study was to ascertain whether, and to what extent, intermittent oscillations in IMP contributed to these well-documented changes in PBF. Interestingly, in the group exposed to compression pressures of $150 \mathrm{mmHg}$, PBF in the compressed limb increased by $20 \%$ after 4 weeks, a magnitude that is remarkably similar to that previously reported (Alomari et al. 2001; Green et al. 1994). However, although the changes in PVC in the compressed forearm followed the same pattern of $\mathrm{BF}$, there was no statistical significance between limbs $(P=0.15)$. Given the lack of robust statistical support, especially to changes in PVC, it seems appropriate to suggest that factors other than or in addition to the mechanical influences explored here contribute primarily to the vascular adaptations to chronic handgrip exercise.

Wall shear stress is a powerful modulator of vascular structure and function and recent reports suggest that changes in the shear stress profile evoked by exercise might be the major signal for the enhanced conduit artery endothelial function and resistance vessel remodeling following exercise training in humans (Tinken et al. 2010). The exact characteristics of the shear stimulus that drive these adaptations are still debatable (Laughlin et al. 2008), but it has been proposed that increased blood flow and anterograde shear are necessary for resistance vessel remodeling following a period of handgrip exercise training (Tinken et al. 2010). Further, it is believed that oscillatory shear stress, characterized by high levels of retrograde shear, might impair conduit artery endothelial function (Thijssen et al. 2009). To gain insights into this issue, we examined shear rate profiles in the brachial artery before and during application of compressions (Table 2). This intervention produces a fluctuating shear profile and has been previously employed to study the impact of different shear characteristics on conduit artery FMD (Pyke et al. 2008). During compression treatment, we observed a marked enhanced oscillatory shear profile with robust changes in peak shear rates (antegrade/retrograde), but notably, the mean shear rate was not significantly altered during the interventions in all three groups (Table 2). Coupled with the minor changes in PBF that we observed after the intervention, these results indicate that increases in mean shear may be necessary for resistance vessel remodeling after exercise in the human forearm (Tinken et al. 2010). Furthermore, the lack of change in FMD in spite of increased oscillatory shear rate during the intervention argues against the notion that oscillatory shear is neither advantageous nor detrimental to conduit artery endothelial function in humans when applied acutely over 4 weeks, as we and others have previously suggested (Green et al. 2005; Laughlin et al. 2008; Newcomer et al. 2008; Thijssen et al. 2009).

\section{Limitations}

One potential limitation of the present study is that we did not include a handgrip exercise training group to verify whether an exercise program with exercise bouts that produce 
changes in IMP similar to the compression protocol employed herein would indeed promote the established vascular adaptations to this type of localized exercise. However, as emphasized above, our study design was based on a robust body of evidence that suggests that handgrip exercise training for 4 weeks evokes a progressive increase in forearm peak blood flow following ischemia that is evident after as little as 1 week (Allen et al. 2003; Alomari et al. 2010; Alomari and Welsch 2007; Franke et al. 1998; Green et al. 1994; Sinoway et al. 1987; Tinken et al. 2010). Given that we used a similar protocol (Franke et al. 1998; Sinoway et al. 1987), methodological approach to study blood flow (Tinken et al. 2010) and a sample size that is comparable to what has been previously used in studies that reported a significant increase in BFC following 4 weeks of handgrip training (Franke et al. 1998; Sinoway et al. 1987), it is reasonable to assume that the protocol employed in this study provides a rigorous test of the hypothesis we advanced.

\section{Conclusions}

The present study is the first to investigate the effects of long-term application of intermittent external forearm compressions on BFC in humans. We observed that repeated mechanical compressions of up to $150 \mathrm{mmHg}$ do not evoke important changes in PBF and $\mathrm{PVC}$ following forearm ischemia. This finding implies that during handgrip exercise, mechanisms other than oscillations in IMP trigger the vascular adaptations that culminate in increased BFC. Nonetheless, future studies are needed to verify whether higher compression pressures can induce more robust and significant effects on BFC in humans. Also, more information is necessary on the effects of this intervention on different vasculatures (e.g., leg), different populations, such as old individuals, and in clinical conditions associated with peripheral vascular rarefaction.

\section{Supplementary Material}

Refer to Web version on PubMed Central for supplementary material.

\section{Acknowledgments}

The authors would like to thank Dr. Richard Madsen for assistance with statistical analysis. This research was supported by HL36088 and a Doctoral Student Research Grant from the American College of Sports Medicine Foundation (B.R.). Bruno Roseguini is a Fulbright/Brazilian Ministry of Education (Capes) fellow.

\section{References}

Allen JD, Geaghan JP, Greenway F, Welsch MA. Time course of improved flow-mediated dilation after short-term exercise training. Med Sci Sports Exerc. 2003; 35:847-853. [PubMed: 12750596]

Alomari MA, Welsch MA. Regional changes in reactive hyperemic blood flow during exercise training: time-course adaptations. Dyn Med. 2007; 6:1. [PubMed: 17222342]

Alomari MA, Welsch MA, Prisby RD, Lee CM, Wood RH. Modification of forearm vascular function following short-term handgrip exercise training. Int J Sports Med. 2001; 22:361-365. [PubMed: 11510873]

Alomari MA, Mekary RA, Welsch MA. Rapid vascular modifications to localized rhythmic handgrip training and detraining: vascular conditioning and deconditioning. Eur J Appl Physiol. 2010; 109:803-809. [PubMed: 20225082]

Barcroft H, Millen JL. The blood flow through muscle during sustained contraction. J Physiol. 1939; 97:17-31. [PubMed: 16995147]

Brown MD, Hudlicka O. Modulation of physiological angiogenesis in skeletal muscle by mechanical forces: involvement of VEGF and metalloproteinases. Angiogenesis. 2003; 6:1-14. [PubMed: 14517399] 
Chen LE, Liu K, Qi WN, Joneschild E, Tan X, Seaber AV, Stamler JS, Urbaniak JR. Role of nitric oxide in vasodilation in upstream muscle during intermittent pneumatic compression. J Appl Physiol. 2002; 92:559-566. [PubMed: 11796664]

Franke WD, Stephens GM, Schmid PG 3rd. Effects of intense exercise training on endotheliumdependent exercise-induced vasodilatation. Clin Physiol. 1998; 18:521-528. [PubMed: 9818157]

Gray SD, Carlsson E, Staub NC. Site of increased vascular resistance during isometric muscle contraction. Am J Physiol. 1967; 213:683-689. [PubMed: 6036785]

Green DJ. Exercise training as vascular medicine: direct impacts on the vasculature in humans. Exerc Sport Sci Rev. 2009; 37:196-202. [PubMed: 19955869]

Green DJ, Cable NT, Fox C, Rankin JM, Taylor RR. Modification of forearm resistance vessels by exercise training in young men. J Appl Physiol. 1994; 77:1829-1833. [PubMed: 7836206]

Green DJ, Bilsborough W, Naylor LH, Reed C, Wright J, O'Driscoll G, Walsh JH. Comparison of forearm blood flow responses to incremental handgrip and cycle ergometer exercise: relative contribution of nitric oxide. J Physiol. 2005; 562:617-628. [PubMed: 15513940]

Hansen-Smith F, Egginton S, Zhou AL, Hudlicka O. Growth of arterioles precedes that of capillaries in stretch-induced angiogenesis in skeletal muscle. Microvasc Res. 2001; 62:1-14. [PubMed: 11421656]

Heffernan KS, Edwards DG, Rossow L, Jae SY, Fernhall B. External mechanical compression reduces regional arterial stiffness. Eur J Appl Physiol. 2007; 101:735-741. [PubMed: 17849144]

Hirche H, Raff WK, Grun D. The resistance to blood flow in the gastrocnemius of the dog during sustained and rhythmical isometric and isotonic contractions. Pflug Arch. 1970; 314:97-112.

Hudlicka O. Is physiological angiogenesis in skeletal muscle regulated by changes in microcirculation? Microcirculation. 1998; 5:7-23. [PubMed: 9702718]

Kirby BS, Carlson RE, Markwald RR, Voyles WF, Dinenno FA. Mechanical influences on skeletal muscle vascular tone in humans: insight into contraction-induced rapid vasodilatation. J Physiol. 2007; 583:861-874. [PubMed: 17495044]

Kroese AJ. Reactive hyperaemia in the calf of trained and untrained subjects: a study with strain gauge plethysmography. Scand J Clin Lab Invest. 1977; 37:111-115. [PubMed: 616036]

Laughlin MH, Ripperger J. Vascular transport capacity of hindlimb muscles of exercise-trained rats. J Appl Physiol. 1987; 62:438-443. [PubMed: 3558204]

Laughlin MH, Roseguini B. Mechanisms for exercise training-induced increases in skeletal muscle blood flow capacity: differences with interval sprint training versus aerobic endurance training. J Physiol Pharmacol. 2008; 59(Suppl 7):71-88. [PubMed: 19258658]

Laughlin MH, Newcomer SC, Bender SB. Importance of hemodynamic forces as signals for exerciseinduced changes in endothelial cell phenotype. J Appl Physiol. 2008; 104:588-600. [PubMed: 18063803]

Lind AR, Williams CA. The control of blood flow through human forearm muscles following brief isometric contractions. J Physiol. 1979; 288:529-547. [PubMed: 469732]

Newcomer SC, Leuenberger UA, Hogeman CS, Handly BD, Proctor DN. Different vasodilator responses of human arms and legs. J Physiol. 2004; 556:1001-1011. [PubMed: 14990681]

Newcomer SC, Sauder CL, Kuipers NT, Laughlin MH, Ray CA. Effects of posture on shear rates in human brachial and superficial femoral arteries. Am J Physiol Heart Circ Physiol. 2008; 294:H1833-H1839. [PubMed: 18245564]

Padilla J, Sheldon RD, Sitar DM, Newcomer SC. Impact of acute exposure to increased hydrostatic pressure and reduced shear rate on conduit artery endothelial function: a limb-specific response. Am J Physiol Heart Circ Physiol. 2009; 297:H1103-H1108. [PubMed: 19633210]

Padilla J, Young CN, Simmons GH, Deo SH, Newcomer SC, Sullivan JP, Laughlin MH, Fadel PJ. Increased muscle sympathetic nerve activity acutely alters conduit artery shear rate patterns. Am J Physiol Heart Circ Physiol. 2010; 298:H1128-H1135. [PubMed: 20154260]

Prior BM, Lloyd PG, Yang HT, Terjung RL. Exercise-induced vascular remodeling. Exerc Sport Sci Rev. 2003; 31:26-33. [PubMed: 12562167]

Prior BM, Yang HT, Terjung RL. What makes vessels grow with exercise training? J Appl Physiol. 2004; 97:1119-1128. [PubMed: 15333630] 
Pyke KE, Poitras V, Tschakovsky ME. Brachial artery flow-mediated dilation during handgrip exercise: evidence for endothelial transduction of the mean shear stimulus. Am J Physiol Heart Circ Physiol. 2008; 294:H2669-H2679. [PubMed: 18408123]

Radegran G, Saltin B. Muscle blood flow at onset of dynamic exercise in humans. Am J Physiol. 1998; 274:H314-H322. [PubMed: 9458882]

Reading JL, Goodman JM, Plyley MJ, Floras JS, Liu PP, McLaughlin PR, Shephard RJ. Vascular conductance and aerobic power in sedentary and active subjects and heart failure patients. J Appl Physiol. 1993; 74:567-573. [PubMed: 8458771]

Reneman RS, Slaaf DW, Lindbom L, Tangelder GJ, Arfors KE. Muscle blood flow disturbances produced by simultaneously elevated venous and total muscle tissue pressure. Microvasc Res. 1980; 20:307-318. [PubMed: 7207224]

Roseguini BT, Mehmet Soylu S, Whyte JJ, Yang HT, Newcomer S, Laughlin MH. Intermittent pneumatic leg compressions acutely upregulate VEGF and MCP-1 expression in skeletal muscle. Am J Physiol Heart Circ Physiol. 2010; 298:H1991-H2000. [PubMed: 20348224]

Saltin B. The interplay between peripheral and central factors in the adaptive response to exercise and training. Ann N Y Acad Sci. 1977; 301:224-231. [PubMed: 270918]

Sejersted OM, Hargens AR. Intramuscular pressures for monitoring different tasks and muscle conditions. Adv Exp Med Biol. 1995; 384:339-350. [PubMed: 8585463]

Sejersted OM, Hargens AR, Kardel KR, Blom P, Jensen O, Hermansen L. Intramuscular fluid pressure during isometric contraction of human skeletal muscle. J Appl Physiol. 1984; 56:287-295. [PubMed: 6706739]

Sinoway LI, Shenberger J, Wilson J, McLaughlin D, Musch T, Zelis R. A 30-day forearm work protocol increases maximal forearm blood flow. J Appl Physiol. 1987; 62:1063-1067. [PubMed: 3571063]

Snell PG, Martin WH, Buckey JC, Blomqvist CG. Maximal vascular leg conductance in trained and untrained men. J Appl Physiol. 1987; 62:606-610. [PubMed: 3558219]

Thijssen DH, Dawson EA, Tinken TM, Cable NT, Green DJ. Retrograde flow and shear rate acutely impair endothelial function in humans. Hypertension. 2009; 53:986-992. [PubMed: 19380611]

Tinken TM, Thijssen DH, Hopkins N, Dawson EA, Cable NT, Green DJ. Shear stress mediates endothelial adaptations to exercise training in humans. Hypertension. 2010; 55:312-318. [PubMed: 20048193] 

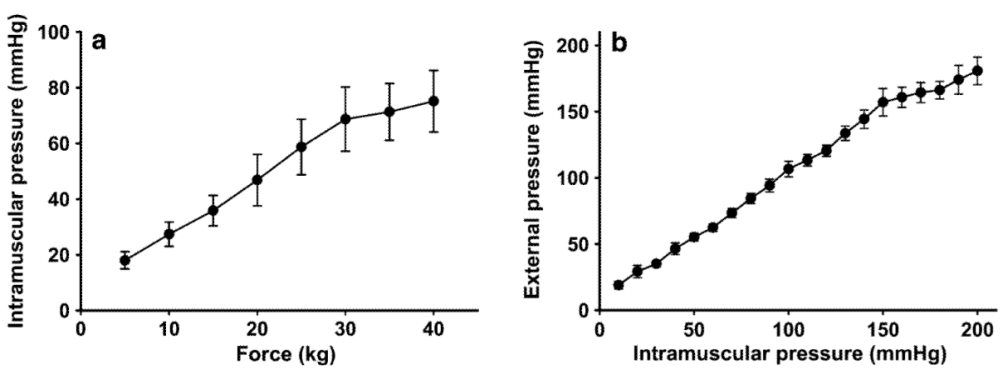

Fig. 1.

Intramuscular pressures (IMP) in the anterior superficial compartment of the forearm during handgrip exercise of various intensities (a) and during application of external pressure (b). Values are as mean $\pm \mathrm{SE}$ 

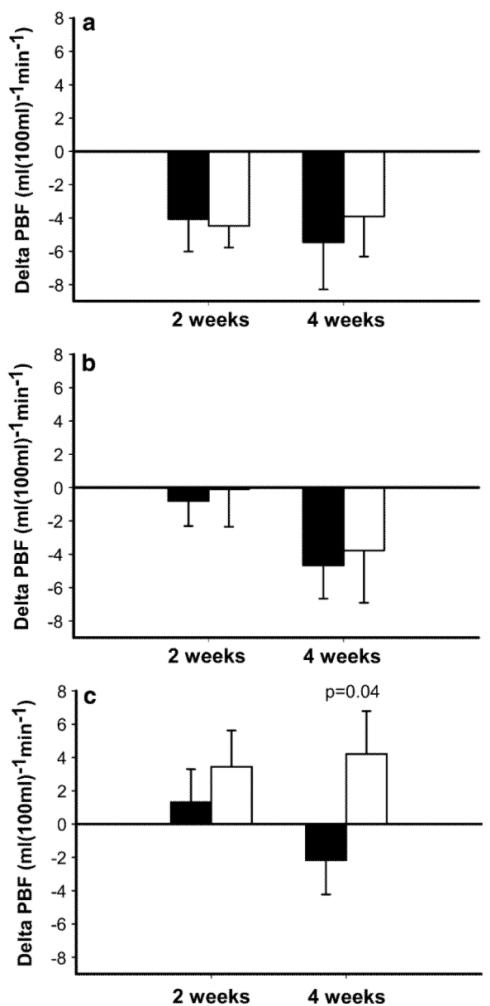

Fig. 2.

Peak blood flow (PBF) changes from the baseline evaluation in the dominant (black bars) and non-dominant forearm (open bars). a $50 \mathrm{mmHg}(n=5)$, b $100 \mathrm{mmHg}(n=8)$ and $\mathbf{c} 150$ $\mathrm{mmHg}$. Comparison between forearms revealed a trend $(P=0.04)$ for increased PBF in the $150 \mathrm{mmHg}$ group after 4 weeks of intervention 


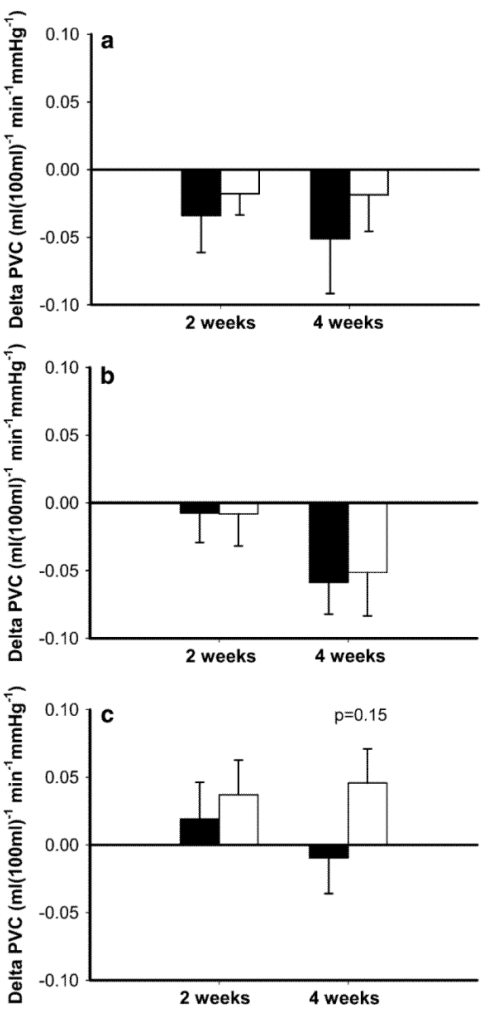

Fig. 3.

Peak vascular conductance (PVC) changes from the baseline evaluation in the dominant (black bars) and non-dominant forearm (open bars). a $50 \mathrm{mmHg}(n=5), \mathbf{b} 100 \mathrm{mmHg}(n=$ 8) and c $150 \mathrm{mmHg}$ 


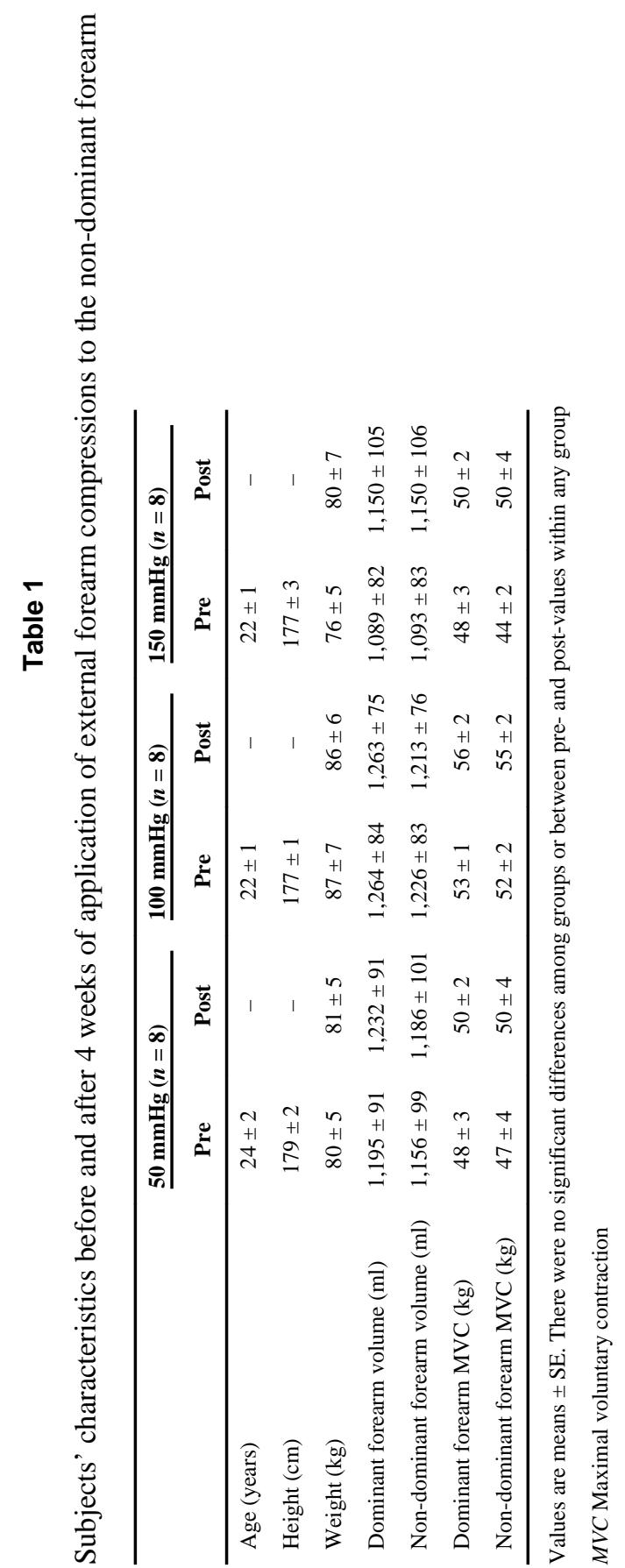




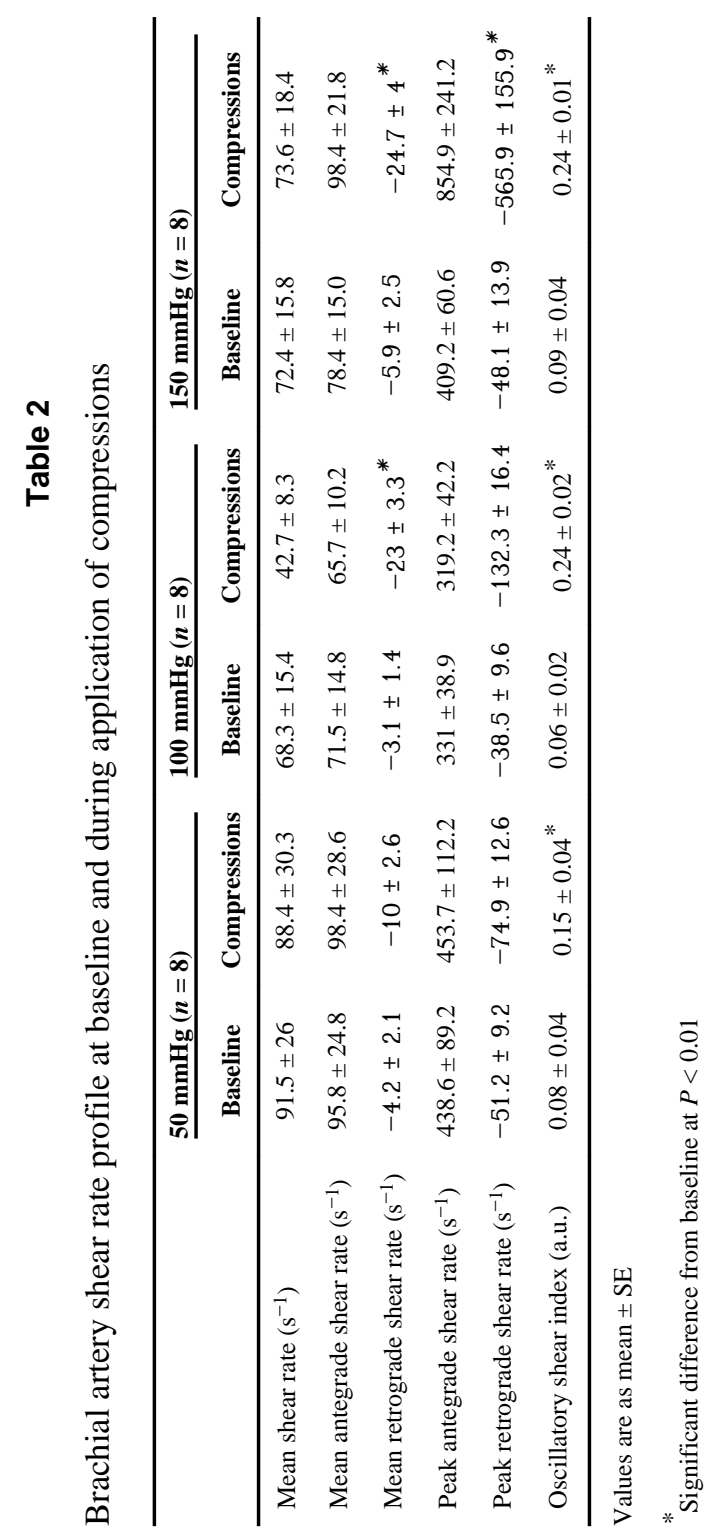




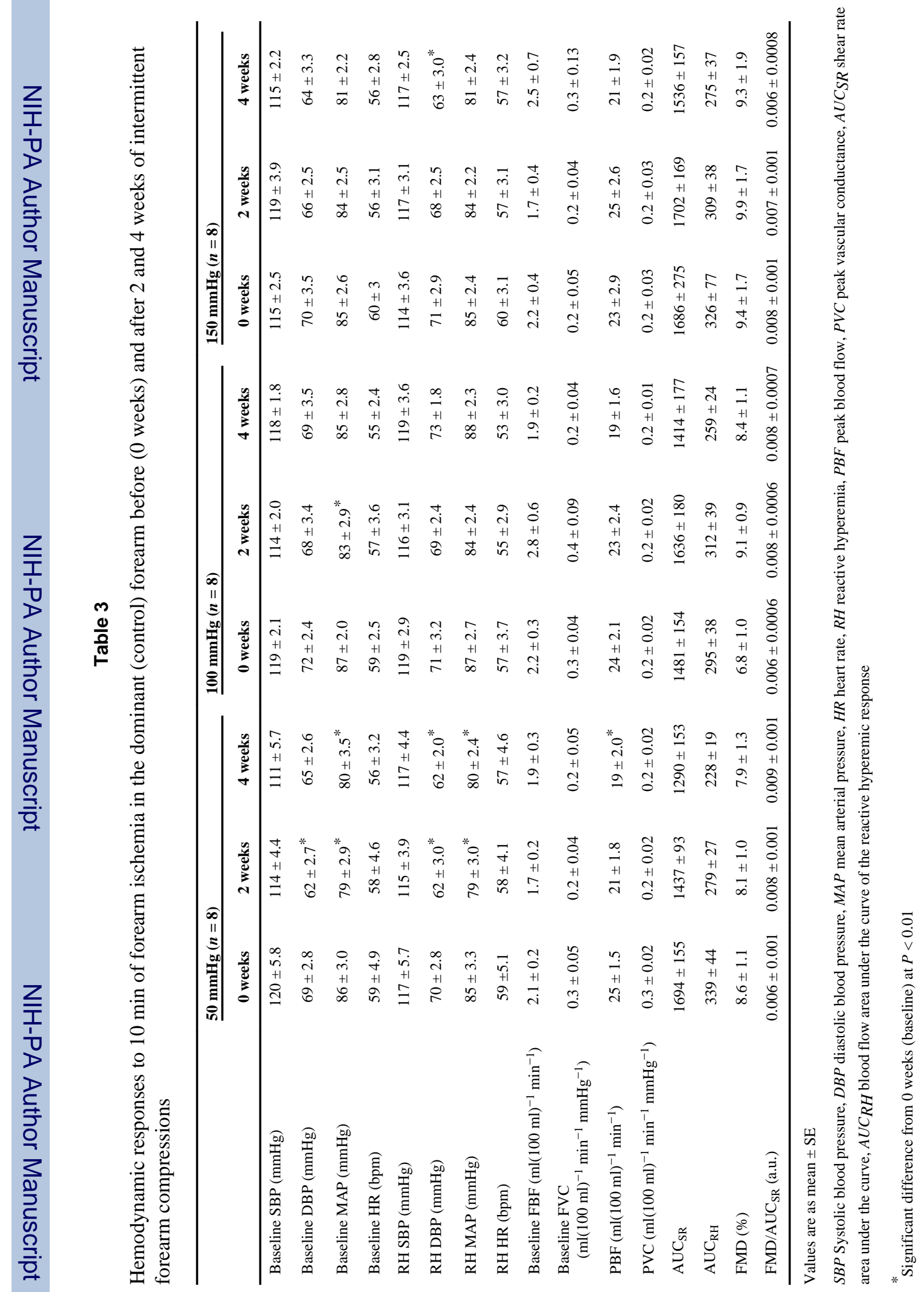




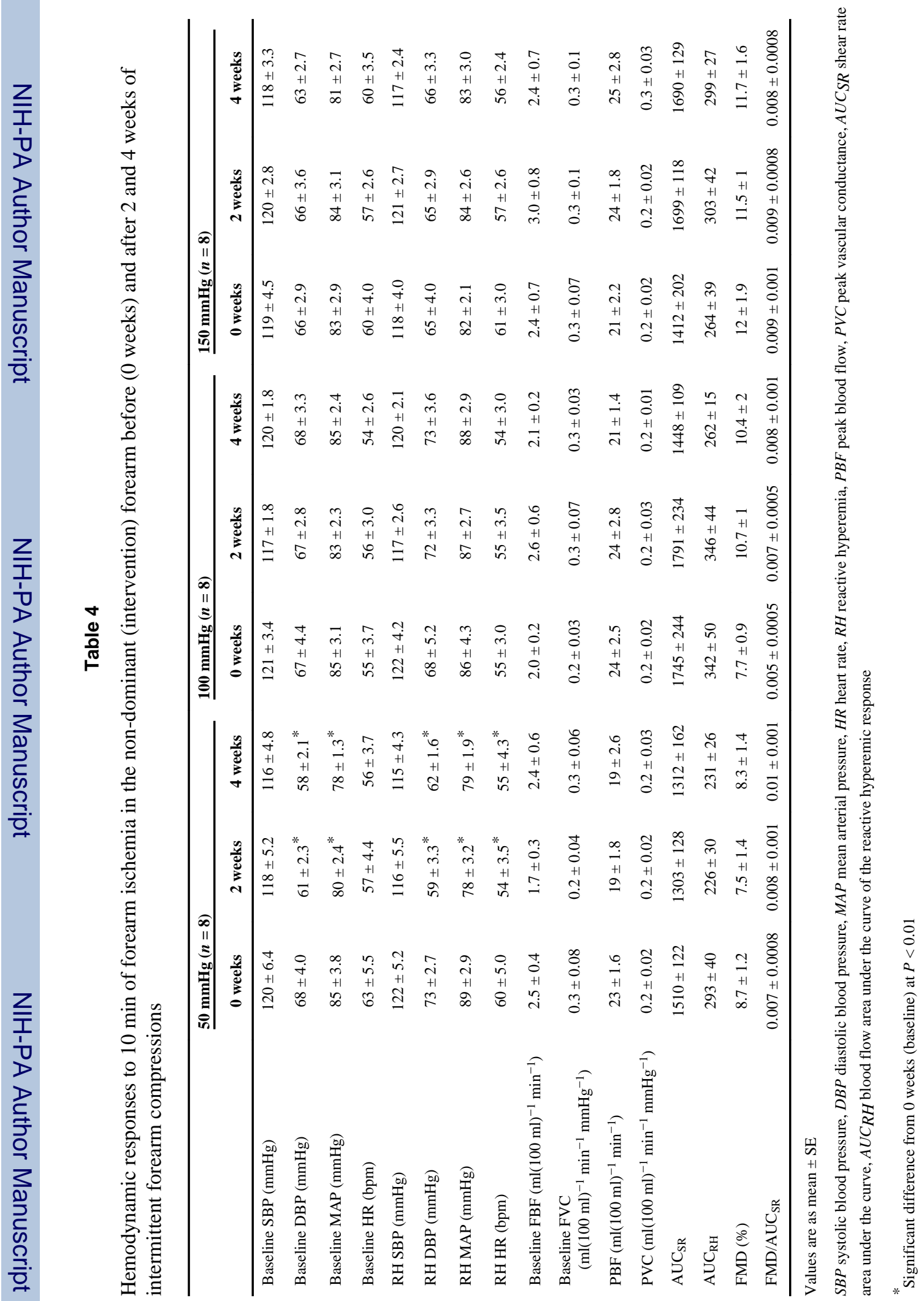

